\title{
Gene profile analysis of osteoblast genes differentially regulated by histone deacetylase inhibitors
}

\author{
Tania M Schroeder ${ }^{1}$, Aswathy K Nair ${ }^{1}$, Rodney Staggs ${ }^{2}$, Anne- \\ Francoise Lamblin ${ }^{2}$ and Jennifer J Westendorf*2,3
}

Address: ${ }^{1}$ Graduate Program in Biochemistry, Molecular Biology and Biophysics, University of Minnesota, 420 Delaware Street SW, Minneapolis, MN, USA, ${ }^{2}$ The Cancer Center, and Department of Orthopaedic Surgery, University of Minnesota, MMC 806, 420 Delaware Street SW, Minneapolis, MN, USA and ${ }^{3}$ Department of Orthopedic Surgery, Mayo Clinic, 200 First Street SW, Rochester, MN, USA

Email: Tania M Schroeder - tania.schroeder@ammd.com; Aswathy K Nair - nairx013@umn.edu; Rodney Staggs - stagg004@umn.edu; AnneFrancoise Lamblin - lambl001@tc.umn.edu; Jennifer J Westendorf* - westendorf.jennifer@mayo.edu

* Corresponding author

Published: 9 October 2007

BMC Genomics 2007, 8:362 doi:10.1/86/|47|-2/64-8-362
Received: 5 April 2007

Accepted: 9 October 2007

This article is available from: http://www.biomedcentral.com/I47I-2/64/8/362

(c) 2007 Schroeder et al; licensee BioMed Central Ltd.

This is an Open Access article distributed under the terms of the Creative Commons Attribution License (http://creativecommons.org/licenses/by/2.0), which permits unrestricted use, distribution, and reproduction in any medium, provided the original work is properly cited.

\begin{abstract}
Background: Osteoblast differentiation requires the coordinated stepwise expression of multiple genes. Histone deacetylase inhibitors (HDIs) accelerate the osteoblast differentiation process by blocking the activity of histone deacetylases (HDACs), which alter gene expression by modifying chromatin structure. We previously demonstrated that HDIs and HDAC3 shRNAs accelerate matrix mineralization and the expression of osteoblast maturation genes (e.g. alkaline phosphatase, osteocalcin). Identifying other genes that are differentially regulated by HDls might identify new pathways that contribute to osteoblast differentiation.

Results: To identify other osteoblast genes that are altered early by HDIs, we incubated MC3T3-EI preosteoblasts with HDIs (trichostatin A, MS-275, or valproic acid) for 18 hours in osteogenic conditions. The promotion of osteoblast differentiation by HDIs in this experiment was confirmed by osteogenic assays. Gene expression profiles relative to vehicle-treated cells were assessed by microarray analysis with Affymetrix GeneChip 4302.0 arrays. The regulation of several genes by HDIs in MC3T3-EI cells and primary osteoblasts was verified by quantitative real-time PCR. Nine genes were differentially regulated by at least two-fold after exposure to each of the three HDIs and six were verified by PCR in osteoblasts. Four of the verified genes (solute carrier family 9 isoform 3 regulator I (Slc9a3rl), sorbitol dehydrogenase $\mathrm{I}$, a kinase anchor protein, and glutathione S-transferase alpha 4) were induced. Two genes (proteasome subunit, beta type 10 and adaptor-related protein complex AP-4 sigma I) were suppressed. We also identified eight growth factors and growth factor receptor genes that are significantly altered by each of the HDIs, including Frizzled related proteins I and 4, which modulate the Wnt signaling pathway.

Conclusion: This study identifies osteoblast genes that are regulated early by HDIs and indicates pathways that might promote osteoblast maturation following HDI exposure. One gene whose upregulation following HDI treatment is consistent with this notion is SIc9a3rl. Also known as NHERFI, $\mathrm{Slc9a3rl}$ is required for optimal bone density. Similarly, the regulation of $\mathrm{Wnt}$ receptor genes indicates that this crucial pathway in osteoblast development is also affected by HDls. These data support the hypothesis that HDls regulate the expression of genes that promote osteoblast differentiation and maturation.
\end{abstract}




\section{Background}

Histone deacetylases (HDACs) and histone acetyltransferases participate in chromatin remodeling and the regulation of gene expression. The opposing activities of these enzymes alter chromatin structure by either adding or removing acetyl groups from lysines in the amino-terminal tails of histones. The addition of acetyl groups to histones by acetyltransferases leads to the recruitment of coactivators and the relaxation of chromatin conformation that is necessary for transcriptional activation [1,2]. Conversely, removal of acetyl groups by HDACs results in a condensed chromatin structure that is restrictive to transcription. Mammalian HDACs are organized into four classes. Class I HDACs $(1,2,3$, and 8) display nuclear localization and ubiquitous tissue expression $[3,4]$. Class II HDACs $(4,5,6,7,9$, and 10) exhibit tissue specific patterns of expression, shuttle between the nucleus and cytoplasm, and are larger than class I HDACs [5]. Class III HDACs (Sirt1-7) require the coenzyme NAD ${ }^{+}$for enzymatic activity [6]. HDAC11 is the sole member of the new Class IV [4].

HDAC inhibitors (HDIs) broadly compromise the activities of class I, II and IV HDACs, albeit with varying efficiencies [7-9]. Natural and synthetic HDIs are divided into several structurally diverse classes including hydroxamic acids such as trichostatin A (TSA), short chain fatty acids such as valproic acid (VPA) and sodium butyrate (NaB), and benzamides such as MS-275 [10]. HDIs inhibit HDAC activity by blocking a channel that leads to the active site and a catalytic zinc ion [11]. In transformed cells, HDIs induce growth arrest, apoptosis, and/or differentiation via many mechanisms $[7,10]$. HDIs are currently in clinical trials as anticancer agents $[10,12]$; they are also established antiepileptic drugs [13] and potential treatments for inflammatory and cardiac diseases $[14,15]$. There are comparatively fewer data on the effects of HDIs on normal cells; however, the existing evidence suggests that normal cells are resistant to the anti-proliferation, pro-apoptosis and pro-differentiation effects of HDIs because their cell cycle checkpoints are intact $[16,17]$.

We previously demonstrated that concentrations of TSA, MS-275 and VPA that were sufficient to induce histone H3 hyperacetylation in primary and MC3T3-E1 osteoblasts modestly increased cell proliferation and viability but had no effect on cell cycle progression [18]. More strikingly, HDIs accelerated the osteoblast maturation process by several days. Thus, short-term exposure to TSA accelerated the appearance of alkaline phosphatase activity and matrix mineralization as well as expression of type I collagen, osteopontin, bone sialoprotein, and osteocalcin genes in MC3T3-E1 cell cultures [18]. TSA, MS-275 and $\mathrm{NaB}$ also increased alkaline phosphatase activity in calvarial organ cultures [18]. Other studies showed that HDIs increase expression of genes associated with osteoblast maturation [19-22], enhance mineralization [21], block glucocorticoid-induced cell cycle arrest in osseous cells [23], and stimulate osteoblast differentiation of multipotent mesenchymal cells $[24,25]$. Suppression of HDAC1 or HDAC3 by RNA interference also accelerated osteoblast maturation $[22,26]$. These results suggest that the gene expression changes occur upon inhibition of HDACs and promote osteoblast terminal differentiation. In this study, we used an unbiased approach to identify osteoblast genes that are altered by HDIs within 18 hours to obtain a better understanding of the early pathways involved in accelerating the osteogenic phenotype.

\section{Results \\ HDIs increase alkaline phosphatase activity during osteoblast differentiation}

We previously demonstrated that HDIs accelerate and enhance alkaline phosphatase expression by MC3T3-E1 pre-osteoblasts after three days. By the second week, the HDI-exposed cultures expressed higher levels of genes associated with osteoblast differentiation (e.g. bone siaoloprotein, osteopontin and osteocalcin) than control cells [18]. The goal of this study was to identify genes that are affected early (within the first 18 hours) by several HDIs because they are more likely to be initiators or early regulators of the process. To demonstrate the differentiation potential of the HDI and vehicle-treated cells used in the microarray experiment, cells in parallel cultures were allowed to differentiate for up to seven days. During the differentiation process, lysates were taken at days $0,1,4$, and 7 and alkaline phosphatase activity was measured. In the DMSO-treated cells, alkaline phosphatase activity increased steadily over time demonstrating that the cells were differentiating appropriately (Figure 1). Alkaline phosphatase activity also increased in the HDI-treated cells and was generally higher in HDI-exposed cells relative to vehicle-treated cells at days 4 and 7 (Figure 1). These results demonstrate that the MC3T3 cells used for microarray analysis differentiated appropriately and that the HDIs accelerated differentiation as expected from our previous studies [18].

\section{Gene expression profiles of HDI treated cells}

To determine the molecular mechanisms whereby HDIs accelerate osteoblast maturation, we used microarray analysis to compare gene expression changes in MC3T3E1 cells treated with HDIs or its vehicle, dimethylsulfoxide (DMSO). On the basis of our previous studies wherein we examined the expression changes in candidate genes after three days of HDI exposure [18], we hypothesized that HDI treatment at the beginning of differentiation would reprogram gene expression and accelerate the entire differentiation process. To identify the relatively early changes in gene expression that occur in response to 


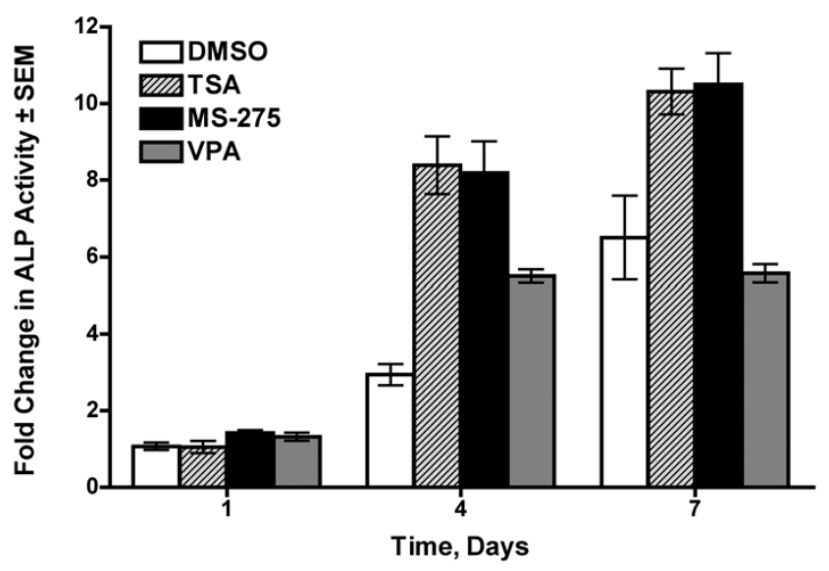

Figure I

HDI treatment accelerates the appearance of alkaline phosphatase activity in differentiating MC3T3 cells. MC3T3 cells were cultured for the indicated times in osteogenic medium. The medium was changed three days with the HDls or vehicle added only at day 0 . Fold change in ALP activity is shown in relation to values obtained at the start of the culture (day 0 ). * denotes a statistically significant change of $p<0.01$ by one-way ANOVA of the HDI-treated sample versus the DMSO-treated sample at that time point.

HDIs in differentiating cells, we isolated RNA from MC3T3-E1 cells cultured in osteogenic medium and HDIs for only 18 hours. Corresponding probes were hybridized to Affymetrix GeneChip arrays and subjected to bioinformatics analyses.

At a false discovery rate (FDR) of 0.01 (99\% confidence) relative to the vehicle (DMSO)-treated cells, 6117 genes were differentially expressed in the TSA-treated cells, 846 genes in the MS-275-treated cells, and 117 genes in the VPA-treated cells. The top 70 TSA-regulated genes, all altered by more than five-fold, are listed in Table 1 . The genes altered more than two-fold by MS-275 and VPA are shown in Tables 2 and 3, respectively. The complete raw data sets have been deposited at the NCBI in the Gene Expression Omnibus data repository (GEO series record GSE9247) [27].

To compare the differentially expressed genes in all three HDI treatments relative to DMSO, Venn analysis was performed (Figure 2). Of the 29 genes differentially regulated by all three HDIs, 21 were induced and eight were suppressed (Table 4). Only four of these 29 genes were differentially regulated more than two-fold by each of the three HDIs (Table 5). Three of these four genes were induced; they are solute carrier family 9 isoform 3 regulator 1 (Slc9a3r1), glutaminyl-peptide cyclotransferase (Qpct), and sorbitol dehydrogenase I (Sdh1). These genes are not affected by TSA in NIH3T3 cells, suggesting specificity to osteoblasts (Figure 3). The suppressed gene is proteasome subunit beta type 10 (Psmb10). This gene was also downregulated by TSA in NIH3T3 cells, indicating that it was not specifically targeted in the osteoblasts. When the FDR was changed to 0.05 ( $95 \%$ confidence), four additional genes were altered more than two-fold by each of the three HDIs (Table 5). Three genes (A kinase anchor protein 12 (Akap12), Glutathione S-transferase alpha 4 (Gsta4), and Ral GEF with a PH domain and $\mathrm{SH}$ binding motif 2 (Ralgps2)) were induced by the HDIs and one gene, Adaptor-related protein complex AP-4 sigma 1 (Ap4s1), was suppressed.

The temporal regulation of several identified genes was determined by quantitative real-time PCRs using mRNAs isolated from independent cultures of MC3T3 cells or primary murine calvarial osteoblasts. Consistent with the GeneChip analysis, TSA was a more potent inducer of Slc9a3r1 than MS-275 or VPA in both MC3T3 cells (Figure 4A) and primary osteoblasts (Figure 4B). Slc9a3r1 induction was detectable at both the RNA and protein levels as early as 4 to 6 hours after HDI exposure (Figure 4B and 4E). Similar effects were observed with Sdh1 mRNA in both MC3T3 (Figure 4C) and primary osteoblasts (Figure 4D) except VPA induced a more rapid increase in Sdh1 expression than TSA. The induction of Akap12 was also confirmed in primary osteoblasts (data not shown). The suppression of Psmb10 and Ap4s1 was confirmed in primary osteoblasts (Figure 5).

\section{Regulation of growth factor and growth factor receptor genes by HDls}

We previously demonstrated that HDIs induced the differentiation rate of MC3T3 cells and primary calvarial osteoblasts [18]. Therefore we analyzed the microarray data sets for growth factor and growth factor receptor genes that were differentially regulated by MS-275, VPA and TSA with greater than $95 \%$ confidence. Of the eight genes identified, only three were induced by all of the HDIs (Table 6). Amphiregulin, a preostoblast growth factor [28], was most consistently and highly increased. Brainderived neurotrophic factor and the Wnt/Norrin receptor, Frizzled 4 (Fz4), were also stimulated by HDIs. In contrast, Fz1, an antagonist of osteoblast growth [29], was suppressed by HDIs. Quantitative real-time PCR analyses verified the differential regulation of several of these genes in primary osteoblasts (Table 6) and MC3T3 cells (data not shown).

\section{Discussion}

HDIs are potential bone anabolic agents due to their ability to promote osteoblast maturation and osteo-progenitor expansion $[18,21,22,24,25]$. We previously showed that the addition of HDIs to MC3T3 cell cultures for three 
Table I: Top 70 known genes altered by TSA in MC3T3-EI cells

\begin{tabular}{|c|c|c|}
\hline Gene & Fold Change & Welch Test \\
\hline \multicolumn{3}{|l|}{ Induced } \\
\hline Ubiquitin carboxy-terminal hydrolase LI & 12.66 & 6.79E-06 \\
\hline Clusterin & 12.58 & I.80E-05 \\
\hline A kinase (PRKA) anchor protein (gravin) 12 & 9.431 & I.06E-07 \\
\hline Abhydrolase domain containing 3 & 9.089 & I.36E-05 \\
\hline Protease, serine, 35 & 9.058 & $3.48 \mathrm{E}-06$ \\
\hline Glutathione S-transferase, alpha 4 & 8.408 & $3.35 \mathrm{E}-08$ \\
\hline Amphiregulin & 7.100 & $2.57 \mathrm{E}-06$ \\
\hline Rab40b, member RAS oncogene family & 6.904 & $1.16 \mathrm{E}-06$ \\
\hline Thioredoxin interacting protein & 6.865 & I.6IE-06 \\
\hline Apolipoprotein L, 2 & 6.604 & 0.001083 \\
\hline Laminin, alpha I & 5.616 & I.70E-04 \\
\hline Solute carrier family 9 , isoform 3 regulator I & 5.565 & $2.32 \mathrm{E}-05$ \\
\hline Growth arrest and DNA-damage-inducible 45 alpha & 5.422 & 3.67E-06 \\
\hline Insulin-like 6 & 5.418 & $6.7 \mathrm{IE}-05$ \\
\hline Thrombospondin I & 5.374 & I.07E-04 \\
\hline Aldehyde dehydrogenase family I, subfamily A7 & 5.359 & 4.73E-04 \\
\hline Erythrocyte protein band 4.1 -like 5 & 5.266 & 6.39E-04 \\
\hline Hemoglobin alpha, adult chain I & 5.119 & $2.93 \mathrm{E}-06$ \\
\hline Glutaminyl-peptide cyclotransferase (glutaminyl cyclase) & 5.046 & $1.22 \mathrm{E}-05$ \\
\hline \multicolumn{3}{|l|}{ Suppressed } \\
\hline SMC4 structural maintenance of chromosomes 4-like I & -5.192 & $5.96 \mathrm{E}-04$ \\
\hline Kinesin family member II & -5.266 & I.07E-08 \\
\hline Lysyl oxidase & -5.284 & I.66E-06 \\
\hline Antigen identified by monoclonal antibody Ki 67 & -5.293 & $3.76 \mathrm{E}-04$ \\
\hline Prostaglandin F receptor & -5.296 & $6.12 \mathrm{E}-04$ \\
\hline Proteasome (prosome, macropain) subunit, beta type 10 & -5.362 & 4.16E-04 \\
\hline Ubiquitin-conjugating enzyme $\mathrm{E} 2 \mathrm{C}$ & -5.393 & 8.64E-06 \\
\hline Cyclin $\mathrm{BI}$, related sequence I & -5.408 & $1.21 \mathrm{E}-05$ \\
\hline Cytoskeleton associated protein 2 & $-5.44 \mid$ & I.47E-07 \\
\hline Polo-like kinase 4 & -5.456 & I.03E-08 \\
\hline Chromodomain helicase DNA binding protein 3 & -5.528 & $6.74 \mathrm{E}-04$ \\
\hline Hyaluronan mediated motility receptor (RHAMM) & -5.547 & $5.4 \mathrm{IE}-04$ \\
\hline Kinesin family member 23 & -5.605 & $2.30 \mathrm{E}-06$ \\
\hline Epithelial membrane protein 3 & -5.606 & $3.65 \mathrm{E}-05$ \\
\hline Proteasome $26 \mathrm{~S}$ subunit, ATPase 3 , interacting protein & -5.623 & 5.0IE-04 \\
\hline Cell division cycle 2 homolog A (S. pombe) & -5.693 & I.43E-05 \\
\hline Formyltetrahydrofolate synthetase domain containing I & -5.725 & $1.96 \mathrm{E}-04$ \\
\hline Nucleolar and spindle associated protein I & -5.780 & I.8IE-05 \\
\hline Centromere autoantigen $A$ & -5.840 & $2.79 \mathrm{E}-04$ \\
\hline Fibroblast growth factor 7 & -5.856 & $6.42 \mathrm{E}-06$ \\
\hline c-Fos induced growth factor & -5.931 & $1.99 \mathrm{E}-04$ \\
\hline High mobility group box 2 & -6.108 & $2.44 \mathrm{E}-08$ \\
\hline Similar to $\mathrm{G} 2 /$ mitotic-specific cyclin BI & -6.166 & I.07E-05 \\
\hline Cyclin A2 & -6.311 & $3.35 \mathrm{E}-08$ \\
\hline Cell division cycle associated 2 & -6.395 & 3.47E-05 \\
\hline Calmodulin-like 4 & -6.400 & 7.64E-04 \\
\hline Heat shock protein, alpha-crystallin-related, B6 & -6.401 & I.69E-04 \\
\hline Mannan-binding lectin serine protease I & -6.413 & $3.19 \mathrm{E}-05$ \\
\hline UDP glycosyltransferase I family, polypeptide A6 & -6.498 & 8.67E-04 \\
\hline Calmodulin binding protein I & -6.501 & $1.68 \mathrm{E}-08$ \\
\hline Aurora kinase $\mathrm{B}$ & -6.531 & $1.74 \mathrm{E}-05$ \\
\hline PDZ binding kinase & -6.548 & $3.16 \mathrm{E}-08$ \\
\hline Minichromosome maintenance deficient 5 & -6.613 & $6.08 \mathrm{E}-08$ \\
\hline Interleukin I receptor-like I & -6.641 & $6.40 \mathrm{E}-08$ \\
\hline RAD5I associated protein I & -6.877 & $9.26 \mathrm{E}-05$ \\
\hline Centromere protein $\mathrm{E}$ & -6.911 & 3.17E-04 \\
\hline F-box only protein 5 & -7.052 & I.79E-07 \\
\hline
\end{tabular}


Table I: Top 70 known genes altered by TSA in MC3T3-EI cells (Continued)

\begin{tabular}{lll}
\hline Serine/threonine kinase 6 & -7.090 & $1.10 \mathrm{E}-07$ \\
Stem cell growth factor & -7.199 & $6.95 \mathrm{E}-05$ \\
Proline 4-hydroxylase, alpha polypeptide III & -7.383 & $3.66 \mathrm{E}-07$ \\
Cell division cycle 20 homolog & -7.469 & $1.27 \mathrm{E}-04$ \\
Asporin & -7.618 & $6.15 \mathrm{E}-04$ \\
Glutathione peroxidase 7 & -8.216 & $1.26 \mathrm{E}-07$ \\
Polo-like kinase I & -8.301 & $9.95 \mathrm{E}-06$ \\
Sushi-repeat-containing protein & -8.701 & $5.38 \mathrm{E}-08$ \\
Ribonucleotide reductase M2 & -9.796 & $2.00 \mathrm{E}-04$ \\
Chromosome condensation I-like & -9.845 & $6.69 \mathrm{E}-04$ \\
Histone I, H2ad & -10.21 & $4.61 \mathrm{E}-06$ \\
Procollagen, type III, alpha I & -10.44 & $6.38 \mathrm{E}-04$ \\
Matrix metalloproteinase I3 & -11.09 & $3.70 \mathrm{E}-05$ \\
Thymidine kinase I & -16.96 & $2.88 \mathrm{E}-05$ \\
\hline
\end{tabular}

All genes were present at a FDR of 0.01 ( $99 \%$ confidence) relative to the vehicle control, DMSO. The list excludes ESTs and other non-annotated sequences.

or six days accelerated the expression of known early and late osteoblast differentiation genes [18]. To identify genes affected early by the HDIs, we differentiated and treated MC3T3-E1 cells with three HDIs or vehicle for 18 hours and analyzed gene expression profiles with Affymetrix microarray gene chips. Relative to vehicle-treated cells, TSA altered the expression of 6117 genes; MS-275 altered 846 genes; and VPA changed 117 genes. The large differences between the numbers of genes affected by the HDIs may be due to differences in their potencies, half-lives and/or specificities. Nanomolar concentrations of TSA inhibit the activities of all HDACs [7]. Likewise, MS-275 is effective at nanomolar concentrations; however, it does not inhibit HDAC8 [9]. In contrast, VPA is effective at millimolar concentrations and specifically inhibits class I and II HDACs with the exceptions of HDAC6 and HDAC10 [8]. Tables 2, 3, 4 list the genes most differentially regulated by each HDI. As indicated in the Venn diagram (Figure 2), some genes on these lists are affected by just one HDI. These genes might appear just in one group because we only assayed one time point; however, it is possible that each HDI affects a distinct subset of genes, some of which might not affect osteoblast differentiation. These differences could affect the suitability of an HDI for a specific application.

All three HDIs (TSA, MS-275 and VPA) used in this study accelerate MC3T3 terminal differentiation and induce alkaline phosphatase activity in calvarial organ cultures [18]. Genes significantly altered by all three HDIs may represent a core group of genes that are responsible for initiating the acceleration of osteoblast differentiation. Of note, well-known osteoblast genes, such as osteocalcin, did not appear in our results. It is likely that the time of analysis (18 hours) was too early to see differential effects on these genes. Of the most differentially regulated genes (Table 5), Slc9a3r1 is the most likely to have roles in osteoblast proliferation and/or differentiation.
Slc9a3r1, also known as $\mathrm{Na}+\mathrm{H}+$ exchanger regulatory factor (NHERF) or ezrin binding protein 50, is an apical membrane phosphoprotein that links membrane proteins with cytoplasmic proteins to regulate actin cytoskeletal reorganization [30]. Slc9a3r1 interacts with numerous signaling proteins [31], including the G-protein coupled receptor for parathyroid hormone [32], and the canonical Wnt signal transducer, $\beta$-catenin [33]; thereby implicating its potentially important role in osteoblast maturation. Slc9a3r1 (NHERF-1) deficient mice develop renal phosphate wasting, but the majority of female mice also had a $25-30 \%$ reduction in bone mineral density and a $40 \%$ decrease in bone mineral content with multiple fractures [34]. We found that overexpression of Slc9a3r1 in osteoblasts by adenoviral transduction was not sufficient to drive osteoblast differentiation (data not shown). These data indicate that NHERF-1 contributes to bone homeostasis but is not sufficient to promote osteoblast maturation.

Another differentially regulated gene is proteasome subunit beta type 10 (Psmb10). Also known as Lmp10 and MECL1, Psmb10 was suppressed by HDIs in both MC3T3 osteoblasts and NIH3T3 cells. Psmb10 is one of ten proteolytically-active beta subunits of the $20 \mathrm{~S}$ core complex within the 26S proteosome [35]. Ubiquitin-mediated proteasomal degradation has an established role in osteoblasts [36,37]. Compounds that bind to the 20S proteasome beta subunits, inhibit the ubiquitin-proteasome pathway and stimulate bone formation in vitro and in vivo [36]. Therefore, the decreased expression of Psmb10 upon HDI treatment is consistent with the observed acceleration of osteoblast differentiation. The specific role HDAC activity plays in the regulation of Psmb10 expression is unclear. The non-specific decreased expression of this gene upon HDI treatment suggests that the Psmb10 promoter is probably not directly regulated 
Table 2: Top 40 known genes altered by MS-275 in MC3T3-EI cells

\begin{tabular}{|c|c|c|}
\hline Gene & Fold Change & Welch Test \\
\hline \multicolumn{3}{|l|}{ Induced } \\
\hline AMP deaminase 3 & 4.177 & $1.93 \mathrm{E}-06$ \\
\hline Musculoskeletal, embryonic nuclear protein I & 3.427 & I.35E-05 \\
\hline Adenylosuccinate synthetase like I & 3.038 & $4.20 \mathrm{E}-05$ \\
\hline Amphiregulin & 2.710 & 4.64E-05 \\
\hline Lysyl oxidase & 2.697 & $1.92 \mathrm{E}-06$ \\
\hline Colony stimulating factor I (macrophage) & 2.694 & $9.32 \mathrm{E}-05$ \\
\hline Fatty acid binding protein 4 , adipocyte & 2.518 & $4.52 \mathrm{E}-05$ \\
\hline Chemokine (C-C motif) ligand 9 & 2.505 & $4.00 \mathrm{E}-05$ \\
\hline Solute carrier family 9 isoform 3 regulator I & 2.477 & $3.63 \mathrm{E}-06$ \\
\hline Sorbitol dehydrogenase I & 2.431 & I.89E-07 \\
\hline Troponin C, cardiac/slow skeletal & 2.408 & $6.23 \mathrm{E}-05$ \\
\hline Proline arginine-rich end leucine-rich repeat & 2.391 & $2.05 \mathrm{E}-05$ \\
\hline Glutaminyl-peptide cyclotransferase (glutaminyl cyclase) & 2.387 & $7.24 \mathrm{E}-05$ \\
\hline Inhibitor of DNA binding I & 2.358 & I.24E-05 \\
\hline Solute carrier family 40 , member I & 2.275 & I.04E-04 \\
\hline Glutathione S-transferase, alpha 4 & 2.268 & $3.40 \mathrm{E}-07$ \\
\hline Septin 4 & 2.195 & $9.94 \mathrm{E}-05$ \\
\hline Voltage-dependent calcium channel gamma subunit-like & 2.187 & $4.78 \mathrm{E}-05$ \\
\hline Immediate early response 3 & 2.171 & $4.22 \mathrm{E}-06$ \\
\hline Dimethylarginine dimethylaminohydrolase I & 2.165 & I.49E-06 \\
\hline Similar to Normal mucosa of esophagus specific gene I & 2.157 & $2.91 \mathrm{E}-06$ \\
\hline MAS-related GPR, member F & 2.122 & I.15E-04 \\
\hline Microtubule-associated protein tau & 2.101 & $9.32 \mathrm{E}-06$ \\
\hline Retinoic acid induced 3 & 2.095 & $1.92 \mathrm{E}-05$ \\
\hline High mobility group AT-hook I & 2.088 & $7.18 \mathrm{E}-05$ \\
\hline A disintegrin-like and metalloprotease with thrombospondin type I motif, 5 & 2.088 & $7.60 \mathrm{E}-05$ \\
\hline Connective tissue growth factor & 2.075 & $3.62 \mathrm{E}-05$ \\
\hline A kinase (PRKA) anchor protein (gravin) 12 & 2.036 & I.78E-05 \\
\hline Aldehyde dehydrogenase family 3 , subfamily AI & 2.029 & I.25E-04 \\
\hline Galactokinase 2 & 2.017 & $2.88 \mathrm{E}-06$ \\
\hline Dystrophia myotonica kinase, $\mathrm{B} 15$ & 2.014 & $3.8 I \mathrm{E}-05$ \\
\hline Thrombospondin I & 2.005 & 7.IIE-05 \\
\hline \multicolumn{3}{|l|}{ Suppressed } \\
\hline Proline 4-hydroxylase, alpha polypeptide III & -2.006 & 4.79E-06 \\
\hline Interleukin I receptor-like I & -2.035 & $4.28 \mathrm{E}-06$ \\
\hline Proteasome (prosome, macropain) subunit, beta type 10 & -2.063 & $2.96 \mathrm{E}-06$ \\
\hline Guanylate nucleotide binding protein 2 & -2.284 & $2.04 \mathrm{E}-06$ \\
\hline Transcription factor AP-2 beta & -2.299 & 2.27E-05 \\
\hline Sirtuin I & -2.450 & $6.2 \mathrm{IE}-05$ \\
\hline Adaptor-related protein complex AP-4, sigma I & -2.484 & I.97E-06 \\
\hline
\end{tabular}

All genes were present at a FDR of 0.01 ( $99 \%$ confidence) relative to the vehicle control, DMSO. The list excludes ESTs and other non-annotated sequences.

by HDACs but rather through a repressor whose expression is sensitive to HDAC activity.

We previously reported that HDIs induce the proliferation and/or survival of MC3T3 cells and primary calvarial osteoblasts but do not affect cell cycle progression [18]. Examination of the microarray gene lists for growth factor and growth factor receptor genes affected by HDIs identified eight genes that could influence osteoblast growth, but only three were increased. Amphiregulin was highly induced by HDIs (Table 6). Amphiregulin binds EGF receptors and is a potent growth factor for preosteoblasts [28]. Brain derived neurotrophic factor mRNA was also increased by HDIs, but its effects on osteoblast growth have not been determined. Two Frizzled genes were also regulated by HDIs. Frizzled- 4 was increased by HDIs, while Frizzled-1 levels were suppressed. Frizzled-4 is a receptor for Norrin [38] and presumably Wnts, which promote osteoblast progenitor proliferation and survival [39]. In contrast, murine Frizzled-1 is a negative regulator of bone formation [29]; thus its suppression is consistent with differentiation promotion by HDIs. No other com- 
Table 3: Top 10 known genes altered by VPA in MC3T3-EI cells

\begin{tabular}{|c|c|c|}
\hline Gene & Fold Change & Welch Test \\
\hline \multicolumn{3}{|l|}{ Induced } \\
\hline Glutathione S-transferase, theta I & 2.345 & I.73E-06 \\
\hline Glutaminyl-peptide cyclotransferase & 2.334 & $2.36 \mathrm{E}-05$ \\
\hline Sorbitol dehydrogenase I & 2.328 & $1.91 \mathrm{E}-06$ \\
\hline Procollagen, type XI, alpha I & 2.216 & 7.84E-06 \\
\hline Solute carrier family 9 , isoform 3 regulator I & 2.203 & $2.33 \mathrm{E}-06$ \\
\hline \multicolumn{3}{|l|}{ Suppressed } \\
\hline Proteasome subunit, beta type 10 & -2.035 & $3.33 \mathrm{E}-06$ \\
\hline $\begin{array}{l}\text { Adaptor-related protein complex AP-4, } \\
\text { sigma I }\end{array}$ & -2.119 & $4.39 \mathrm{E}-07$ \\
\hline RIKEN cDNA $2310039 E 09$ gene & -2.759 & I.48E-05 \\
\hline Tenascin $\mathrm{N}$ & -2.913 & 8.53E-06 \\
\hline Chemokine (C-X-C motif) receptor 6 & -3.728 & I.56E-07 \\
\hline
\end{tabular}

All genes were present at a FDR of 0.01 ( $99 \%$ confidence) relative to the vehicle control, DMSO.

Table 4: Genes differentially regulated by all three HDIs

\begin{tabular}{|c|c|c|c|}
\hline Gene Description & TSA & MS-275 & VPA \\
\hline Induced & \multicolumn{3}{|c|}{ Fold Change } \\
\hline Thioredoxin interacting protein & 6.865 & 1.530 & 1.404 \\
\hline Solute carrier family 9 , isoform 3 regulator I & 5.565 & 2.477 & 2.203 \\
\hline Glutaminyl-peptide cyclotransferase (glutaminyl cyclase) & 5.046 & 2.387 & 2.334 \\
\hline Sorbitol dehydrogenase I & 4.455 & 2.431 & 2.328 \\
\hline Histocompatibility $2, Q$ region locus 5 & 4.009 & 1.634 & 1.820 \\
\hline Phosphomannomutase I & 3.895 & 1.902 & 1.808 \\
\hline RAB3D, member RAS oncogene family & 3.717 & 1.528 & 1.742 \\
\hline $\mathrm{Cbp} / \mathrm{p} 300$-interacting transactivator & 3.458 & 1.658 & 1.321 \\
\hline Protease, serine, 11 & 3.326 & 1.836 & 1.744 \\
\hline Syntaxin II & 2.488 & 1.942 & 1.750 \\
\hline Protein phosphatase I, regulatory (inhibitor) subunit I4c & 2.484 & 1.347 & 1.830 \\
\hline Lysosomal-associated protein transmembrane 4B & 2.366 & 1.503 & 1.637 \\
\hline Similar to normal mucosa of esophagus specific gene I & 2.099 & 2.157 & 1.930 \\
\hline F-box and leucine-rich repeat protein 16 & 2.019 & 1.746 & 1.561 \\
\hline HIV-I Rev binding protein & 1.953 & 1.406 & 1.414 \\
\hline Heat shock 70 kDa protein 5 binding protein I & 1.946 & 1.358 & 1.589 \\
\hline Cysteine rich protein 2 & 1.929 & 1.847 & 1.773 \\
\hline Asparagine synthetase & 1.597 & 1.451 & 1.248 \\
\hline Serum deprivation response & 1.485 & 1.517 & 1.676 \\
\hline Thymus cell antigen I, theta & 1.419 & 1.638 & 1.676 \\
\hline Ribonuclease, RNase A family 4 & 1.341 & 1.341 & 1.529 \\
\hline \multicolumn{4}{|l|}{ Suppressed } \\
\hline Interleukin I receptor-like I & -1.634 & -2.035 & $-1.83 \mid$ \\
\hline Low density lipoprotein receptor & -2.487 & -1.282 & -1.377 \\
\hline Proteolipid protein 2 & -2.717 & -1.274 & -1.233 \\
\hline PHD finger protein 15 & -4.100 & -1.498 & -1.613 \\
\hline Vesicle-associated membrane protein 8 & -4.130 & -1.417 & -1.500 \\
\hline Proteasome (prosome, macropain) subunit, beta type 10 & -5.362 & -2.063 & -2.035 \\
\hline Chromosome condensation I-like & -7.016 & -1.514 & -1.721 \\
\hline Glutathione peroxidase 7 & -8.216 & -1.768 & -1.972 \\
\hline
\end{tabular}

All genes were present at a FDR of 0.01 ( $99 \%$ confidence) relative to the vehicle control, DMSO. 
Table 5: Genes differentially regulated more than two-fold by all three HDIs

\begin{tabular}{|c|c|c|c|}
\hline Gene Description & TSA & MS-275 & VPA \\
\hline Induced & \multicolumn{3}{|c|}{ Fold Change } \\
\hline A kinase anchor protein 12 (Akap I2) & 9.5 & 2.0 & 2.6 \\
\hline Glutaminyl-peptide cyclotransferase (Qpct)* & 5.0 & 2.4 & 2.3 \\
\hline Glutathione S-transferase, alpha 4 (Gsta4) & 8.5 & 2.3 & 3.1 \\
\hline Ral GEF with PH domain and SH3 binding motif 2 (Ralgps2) & 2.2 & 2.0 & 2.1 \\
\hline Solute carrier family 9 , isoform 3 regulator I (Slc9a3rl)* & 5.6 & 2.5 & 2.2 \\
\hline Sorbitol dehydrogenase I (Sdh I)* & 4.5 & 2.4 & 2.3 \\
\hline \multicolumn{4}{|l|}{ Suppressed } \\
\hline Adaptor-related protein complex AP-4, sigma I (Ap4sI) & -4.9 & -2.5 & -2.1 \\
\hline Proteasome (prosome, macropain) subunit, beta type 10 (Psmb I0)* & -5.4 & -2.1 & -2.0 \\
\hline
\end{tabular}

All genes were present at a FDR of 0.05 (95\% confidence) relative to the vehicle control, DMSO. An asterisk $(*)$ indicates genes present at a FDR of 0.01 ( $99 \%$ confidence).

ponents of the Wnt signaling pathway were significantly regulated by all HDIs at the 18-hour time point.

\section{Conclusion}

We identified many osteoblast genes whose expression levels are altered by HDIs. All genes we have tested to date are similarly differentially regulated in both MC3T3-E1 cells and primary murine calvarial osteoblasts. These data improve our understanding of how HDIs promote osteoblast differentiation by identifying genes that are altered within the first 18 hours of HDAC inhibition.

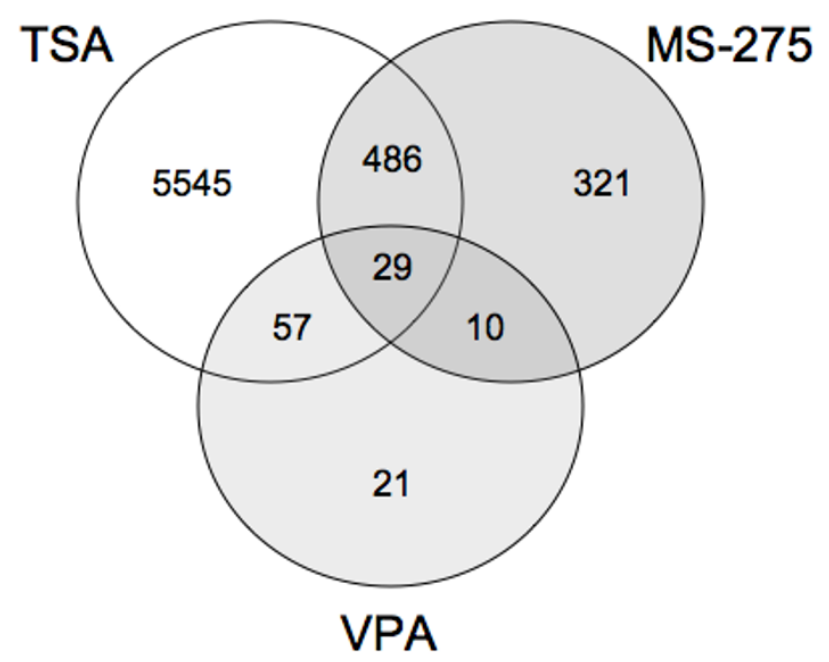

Figure 2

Venn diagram of differentially expressed genes in TSA, MS-275, or VPA-treated cells. MC3T3 cells were treated with an HDI or DMSO for 18 hours. Affymetrix GeneChip microarrays and bioinformatics analysis identified genes that were differentially expressed by more than I.I fold in HDI-treated cells relative vehicle (DMSO)-treated cells with a FDR of 0.01 ( $99 \%$ confidence).

\section{Methods \\ Cell culture}

MC3T3-E1 preosteoblasts were plated at $2 \times 10^{5}$ cells per 6 -cm plate and $4 \times 10^{4}$ cells per well of a 12 -well plate and differentiated in Minimal Essential Medium (Invitrogen, Carlsbad, CA) containing 10\% FBS (Invitrogen and Cambrex Bioscience/Lonza, Basel. Switzerland), $100 \mathrm{U} / \mathrm{ml}$ penicillin, $100 \mu \mathrm{g} / \mathrm{ml}$ streptomycin, $50 \mu \mathrm{g} / \mathrm{ml}$ ascorbic acid, $10 \mathrm{mM} \beta$-glycerol phosphate and one of the following compounds: $20 \mathrm{nM}$ TSA (Sigma, St. Louis, MO), 500 nM MS-275 (Calbiochem, San Diego, CA), 500 mM VPA (Sigma), or DMSO (vehicle). During the differentiation assay, the osteogenic medium was replaced every three days with the HDIs or vehicle added only at day 0. C2C12 cells and NIH3T3 fibroblasts were maintained Dulbecco's modified Eagle's medium containing 10\% FBS, $100 \mathrm{U} / \mathrm{ml}$ penicillin and $100 \mu \mathrm{g} / \mathrm{ml}$ streptomycin. Primary calvarial osteoblasts were isolated as previously described [18]. Briefly, calvaria from newborn CD1 mice were collected and sequentially rinsed in Hank's Balanced Salt Solution (Invitrogen) and serum-free Minimal Essential Medium (Invitrogen). Calvaria were digested into a single cell suspension in serum-free alpha- Minimal Essential Medium containing $2 \mathrm{mg} / \mathrm{ml}$ collagenase and $0.25 \%$ trypsin. Cells were washed, plated at $2 \times 10^{5}$ cells $/ 10 \mathrm{~cm}$ plate and incubated with HDI as described above.

\section{RNA, cDNA, and biotin-labeled cRNA preparation}

To ensure statistical significance of microarray analyses, quadruplicate cultures of MC3T3-E1 cells were incubated in osteogenic medium containing DMSO or HDIs. Total RNA was isolated from each MC3T3-E1 cell culture and primary calvarial osteoblasts with Trizol reagent (Invitrogen). For preparation of GeneChip samples, biotinlabeled cRNA was prepared from each of the quadruplicate cultures according to the Affymetrix protocol. Briefly, RNA was denatured at $70^{\circ} \mathrm{C}$ with T7-oligo (dT) primer and reverse transcribed using Superscript II at $42^{\circ} \mathrm{C}$ for 1 hour. Second strand cDNA synthesis was performed by 

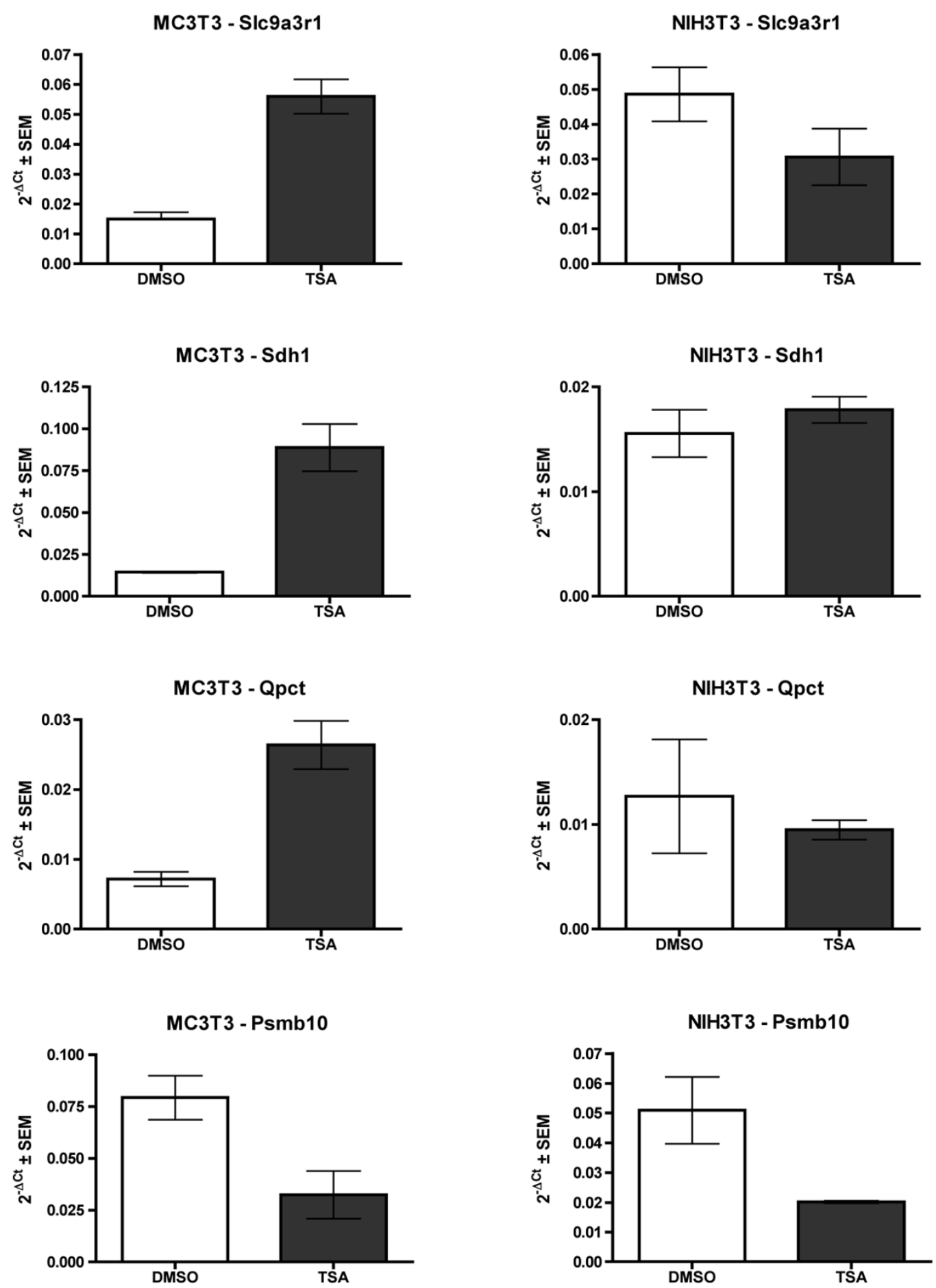

Figure 3

Specificity of HDI-inducible genes. MC3T3 and NIH3T3 cells were cultured in osteogenic medium containing TSA or DMSO for 18 hours. mRNAs were isolated, reverse transcribed and amplified using semi-quantitative real-time PCR with primers for Slc9a3rl (A) or Sdh I (B), Qpct (C) and PsmbIO (D). Comparative threshold values represent the mean of three samples normalized to actin levels. 

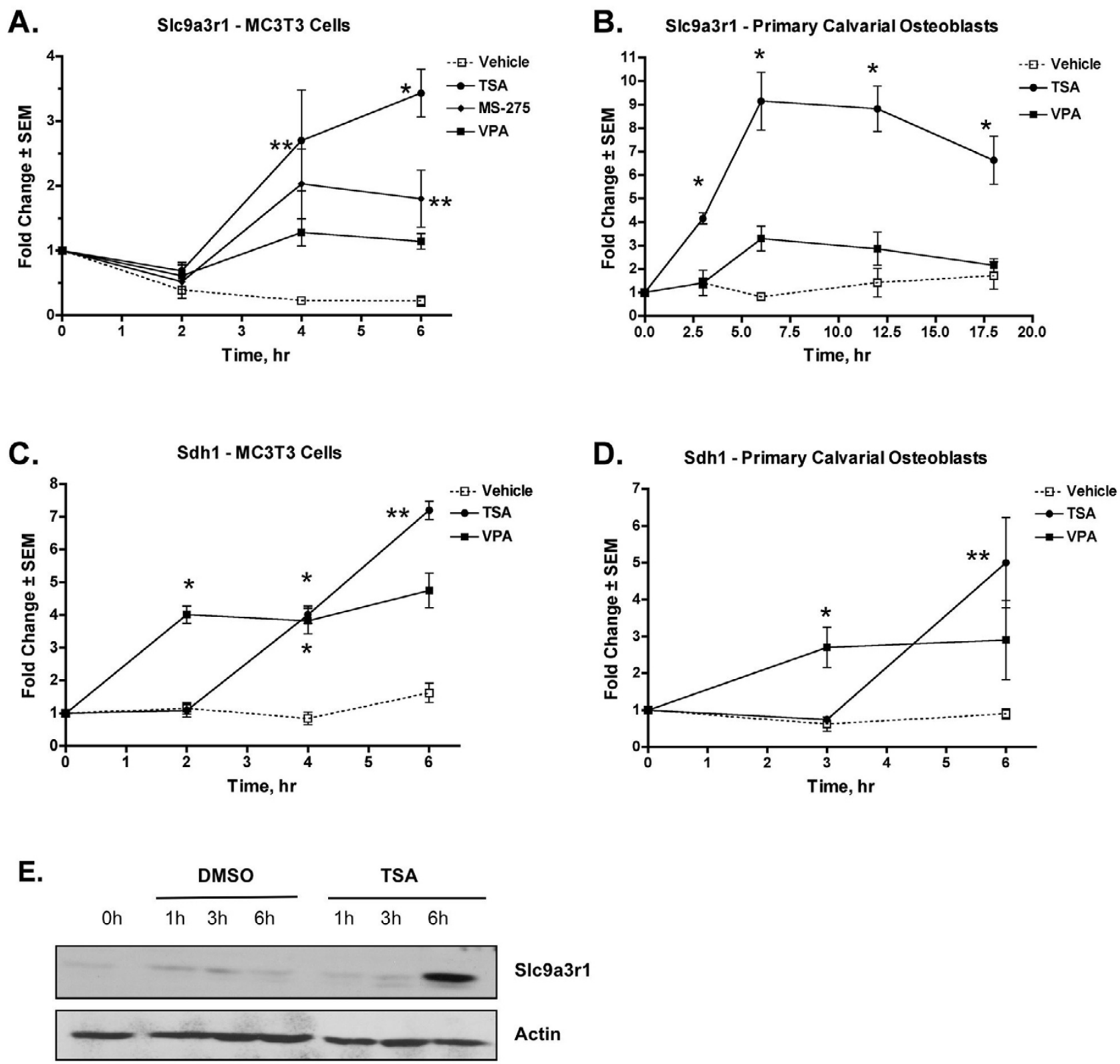

Figure 4

Verification of SIc9a3rI and Sdh I as HDI-inducible genes. MC3T3 (A and C) or primary calvarial osteoblasts (B and D) were cultured in osteogenic medium containing the indicated HDI or DMSO. mRNAs were isolated at various times over a 6 or 18 hour period and subjected to quantitative real-time PCR with primers for Slc9a3rI (A and B) or Sdh I (C and D). Values are relative to those obtained from DMSO-treated samples at each time point and represent the mean of three samples. For (A-D), * denotes a statistically significant change of $p<0.01$ and ** denotes $p<0.05$ by one-way ANOVA of the HDI-treated sample versus the DMSO-treated sample at that time point. (E) $\mathrm{C} 2 \mathrm{Cl} 2$ cells were cultured in osteogenic medium and $20 \mathrm{nM}$ TSA for the indicated times. Slc9a3r I protein levels were determined by immunoblotting.

incubating first strand cDNA with Escherichia coli DNA polymerase I, E. coli. DNA ligase, RNase H, and dNTPs for 2 hours at $16^{\circ} \mathrm{C}$. Biotin-labeled cRNA was prepared from double-stranded cDNA using the Affymetrix GeneChip IVT Labeling kit (Affymetrix, Santa Clara, CA), then puri- fied and fragmented using the Affymetrix Sample Cleanup Module. 

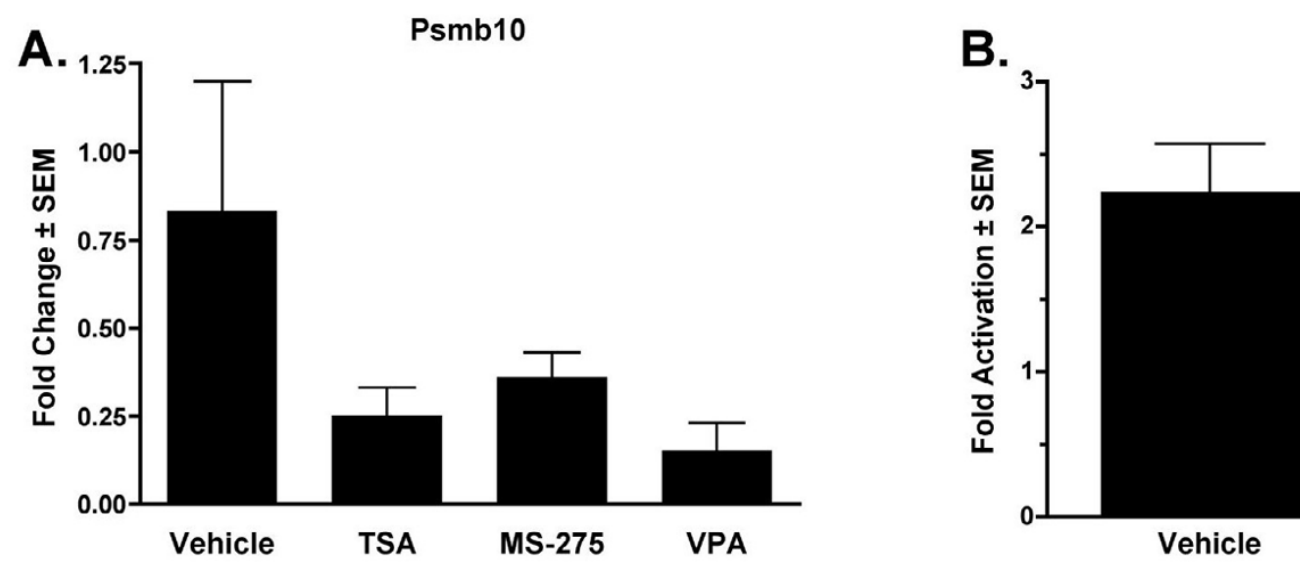

Ap4s1

Figure 5

Verification of Psmb I 0 and Ap4s I as HDI-suppressed genes. Primary calvarial osteoblasts were cultured in osteogenic medium containing the indicated HDI or DMSO. mRNAs were isolated after 18 hours and subjected to quantitative real-time PCR with primers for PsmbI0 (A) or Ap4sI (B). Values are relative to those obtained from DMSO-treated samples at each time point and represent the mean of three samples. ** denotes $p<0.05$ by one-way ANOVA of the HDI-treated sample versus the DMSO-treated sample at that time point.

\section{Affymetrix genechip}

Hybridization of the biotinylated-cRNA to the Affymetrix GeneChip Mouse Genome 430 2.0 Array was performed by the BioMedical Genomics Center's microarray facility at the University of Minnesota using Affymetrix Genechip ${ }^{\circledast}$ Hybridization Oven 640 and Fluidics Station 450. For each condition, four chips were hybridized. Scanning of the chips to detect and quantify hybridization signals was done with the Affymetrix Genechip ${ }^{\circledR}$ Scanner 3000. The gene expression data were preprocessed and analyzed by the University of Minnesota Cancer Center Informatics Shared Resource. Raw data measurements were imported into Genedata Expressionist ${ }^{\circledR}$ Refiner (EPro1.0.32) for overall chip hybridization quality assessment, correction and condensation of probe sets intensity values. Robust
Multichip Average (RMA) method was applied for global background subtraction and cross array normalization [40]. Processed expression data were imported into Genedata Expressionist Analyst. General assessment of the data distribution was performed with Principle Component Analysis, boxplot and log-log data plots. Following LOESS normalization, differential expression measures were calculated by Welch test comparisons of control DMSO (vehicle) versus each HDAC inhibitor. Significant genes from each comparison were selected using the BenjaminiHochberg method to control for a maximum false discovery rate (FDR) of $95 \%$ or $99 \%$ as indicated. Values for fold change were calculated and genes demonstrating a net change in gene expression of two fold or greater were selected for further analysis.

Table 6: Growth factor and growth factor receptor genes regulated by HDIs

\begin{tabular}{|c|c|c|c|c|c|}
\hline \multirow[b]{2}{*}{ Growth factor/receptor } & \multicolumn{3}{|c|}{ MC3T3 Cells Microarray } & \multicolumn{2}{|c|}{ Primary Osteoblasts qRT-PCR } \\
\hline & TSA & MS-275 & VPA & TSA & VPA \\
\hline & \multicolumn{5}{|c|}{ Fold Change } \\
\hline Amphiregulin & 7.10 & 2.74 & 1.82 & 24.8 & 2.56 \\
\hline Brain-derived neurotrophic factor & 2.51 & 1.66 & 1.77 & ND & ND \\
\hline Fibroblast growth factor 7 & -5.80 & -1.10 & -1.17 & -29.9 & 1.05 \\
\hline Frizzled I & -2.17 & -1.25 & -1.24 & -2.53 & -1.44 \\
\hline Frizzled 4 & 2.23 & 1.37 & 1.53 & 1.79 & 2.57 \\
\hline Interleukin I receptor-like I & -1.63 & -2.04 & -1.83 & -52.1 & 1.04 \\
\hline Interleukin 17 receptor c & -2.62 & -1.50 & -1.43 & ND & ND \\
\hline Smoothened & -2.60 & -1.53 & -1.17 & ND & ND \\
\hline
\end{tabular}

Cells were incubated with HDls for $18 \mathrm{hr}$. All genes were present at a FDR of 0.05 (95\% confidence) relative to the vehicle control, DMSO. ND, not determined. 
Table 7: Primer sequences used for PCRs

\begin{tabular}{|c|c|c|c|c|}
\hline Gene & Strand & Primer Sequences & Annealing Temp $\left({ }^{\circ} \mathrm{C}\right)$ & Product Length (bp) \\
\hline \multirow[t]{2}{*}{ Akap 12} & $\mathrm{~F}$ & 5' GCCAGTGAAGAACATGAGCAG & 56 & 160 \\
\hline & $\mathrm{R}$ & 5' CTATGCAATCTGCTTTGTCTTGG & & \\
\hline \multirow[t]{2}{*}{ Ap4sI } & $\mathrm{F}$ & 5' GTACTTCAGCCGAGTGAGTG & 56 & 202 \\
\hline & $\mathrm{R}$ & 5' CTCTTCCTTGGCCTTCACAG & & \\
\hline \multirow[t]{2}{*}{ Amphiregulin } & $\mathrm{F}$ & 5' GACTCACAGCGAGGATGACAAG & 56 & 249 \\
\hline & $\mathrm{R}$ & 5' GCTTGGCAATGATTCAAC & & \\
\hline \multirow[t]{2}{*}{ Fibroblast growth factor 7} & $\mathrm{~F}$ & 5' CAGTTTGGAAAGAGCGACGAC & 56 & 170 \\
\hline & $\mathrm{R}$ & 5' GGCAGGATCCGTGTCAGTATC & & \\
\hline \multirow[t]{2}{*}{ Frizzled-I } & $\mathrm{F}$ & 5' CAAGGTTTACGGGCTCATGTAC & 56 & 180 \\
\hline & $\mathrm{R}$ & 5' GTAACAGCCGGACAGGAAAATG & & \\
\hline \multirow[t]{2}{*}{ Frizzled-4 } & $\mathrm{F}$ & 5' CTGCAGCATGCCTAATGAGAG & 56 & 185 \\
\hline & $\mathrm{R}$ & 5' CGTCTGCCTAGATGCAATCA & & \\
\hline \multirow[t]{2}{*}{ Interleukin I receptor-like I } & $\mathrm{F}$ & 5' CCAGCCAGAGTGGAAGACTC & 56.9 & 189 \\
\hline & $\mathrm{R}$ & 5' CAGGTCAATTGTTGGACACG & & \\
\hline \multirow[t]{2}{*}{ Psmblo } & $\mathrm{F}$ & 5' CTTTACTGCCCTTGGCTCTG & 56.9 & 168 \\
\hline & $\mathrm{R}$ & 5' GTGATCACACAGGCATCCAC & & \\
\hline \multirow[t]{2}{*}{ Qpct } & $\mathrm{F}$ & 5' GAGGAGGCACTGAAGGAGTG & 53 & 162 \\
\hline & $\mathrm{R}$ & 5' GAAGATCGGACTGGATGCTC & & \\
\hline \multirow[t]{2}{*}{ SdhI } & $\mathrm{F}$ & 5' CTGCCGATTCTACAAGCACA & 56.9 & 183 \\
\hline & $\mathrm{R}$ & 5' AGCAAAGTGACCATCCCAAC & & \\
\hline \multirow[t]{2}{*}{ Slc9a3rl } & $\mathrm{F}$ & 5' CAAAGTGTCCCCTCACCAGT & 56.9 & 209 \\
\hline & $\mathrm{R}$ & 5' AATGAACCCAAGATGCCAAG & & \\
\hline
\end{tabular}

\section{Quantitative real-time PCR}

Total RNA was isolated from MC3T3-E1, NIH3T3, and primary murine calvarial cells with Trizol reagent (Invitrogen). RNA ( $1 \mu \mathrm{g})$ was reverse transcribed to cDNA with the Invitrogen Superscript Kit. cDNA was amplified with the Qiagen Quantitect SYBR Green RT-PCR kit using gene specific primers (Table 7) in a MyIQ Single Color RealTime PCR Detection System (BioRad). Quantification and normalization to actin amplicons were performed as previously described [26]. Statistical analyses were performed with Prism 4.0 (GraphPad Software, San Diego, CA).

\section{Alkaline phosphatase activity assay}

Alkaline phosphatase activity was measured on the indicated days as previously described [26]. Briefly, MC3T3E1 cells were washed in PBS, lysed in 0.2\% NP-40 and 1 $\mathrm{mM} \mathrm{MgCl}_{2}$, sonicated, and spun at $3000 \mathrm{rpm}$ for $15 \mathrm{~min}$ utes at $4{ }^{\circ} \mathrm{C}$. The supernatants were added to a reaction solution containing 0.6 M 2-amino-2-methyl-1-propanol, $2.4 \mathrm{mM} \mathrm{MgCl}_{2}$, and $9.6 \mathrm{mM}$ p-nitrophenyl phosphate and incubated at $37^{\circ} \mathrm{C}$ for 30 minutes, at which time the reactions were stopped with $2 \mathrm{~N} \mathrm{NaOH}$ and the absorbance read at $410 \mathrm{~nm}$. Alkaline phosphatase activity was normalized to protein content. Protein content was determined using the DC Protein Assay system (BioRad).

\section{Immunoblotting}

Cell lysates were prepared by rinsing cultures with PBS prior to lysing the cells on ice for 5 minutes in modified RIPA buffer (50 mM Tris-HCl, pH 7.4, 150 mM sodium chloride, 1\% NP-40, 0.25\% sodium deoxycholate, $1 \mathrm{mM}$ EDTA) supplemented with complete protease inhibitor tablets (Roche, Basel, CH). Crude lysates were sonicated and cleared by centrifugation at $10,000 \mathrm{rpm}$ at $4^{\circ} \mathrm{C}$. Total protein was quantified using the detergent-compatible protein assay (BioRad, Hercules, CA). Protein $(50 \mu \mathrm{g})$ was resolved by SDS-PAGE on an $8 \%$ gel and transferred to Immobilon-P membranes (Millipore, Billerica, MA). Membranes were sequentially blotted with antibodies against NHERF1/EBP50 (ab3452; Abcam, Cambridge, MA) and HRP-conjugated secondary antibodies (Sigma). Proteins were visualized using ECL-Plus chemiluminescent substrate (G.E. Health Systems).

\section{Competing interests}

The author(s) declares that there are no competing interests.

\section{Authors' contributions}

TMS generated the microarray data and drafted the manuscript. AKN verified differentially expressed genes. RS and AFL performed bioinformatics analysis of the microarray data. JJW provided support, direction, and oversight of the experiments and revised the final manuscript. All authors have read and approved the final manuscript.

\section{Acknowledgements}

This work was supported by grants from the National Institutes of Health (ROIs AR48I47 and AR50938, T32 CA09138). We thank our colleagues, Dr. Paul Marker and Sheri Kuslak, for providing other reagents. We thank Xiaodong Li for technical assistance. 


\section{References}

I. Grunstein M: Histone acetylation in chromatin structure and transcription. Nature 1997, 389(6649):349-352.

2. Peterson CL, Laniel MA: Histones and histone modifications. Curr Biol 2004, I4( I 4):R546-5 I.

3. Gray SG, Ekstrom TJ: The Human Histone Deacetylase Family. Exp Cell Res 200I, 262(2):75-83.

4. Gregoretti IV, Lee YM, Goodson HV: Molecular evolution of the histone deacetylase family: functional implications of phylogenetic analysis. J Mol Biol 2004, 338(I):|7-3I.

5. Yang XJ, Gregoire S: Class II histone deacetylases: from sequence to function, regulation, and clinical implication. Mol Cell Biol 2005, 25(8):2873-2884.

6. Blander G, Guarente L: The Sir2 family of protein deacetylases. Annu Rev Biochem 2004, 73:417-435.

7. Yoshida M, Matsuyama A, Komatsu Y, Nishino N: From discovery to the coming generation of histone deacetylase inhibitors. Curr Med Chem 2003, I 0(22):235I-2358.

8. Gurvich N, Tsygankova OM, Meinkoth JL, Klein PS: Histone deacetylase is a target of valproic acid-mediated cellular differentiation. Cancer Res 2004, 64(3): 1079-1086.

9. Hu E, Dul E, Sung CM, Chen Z, Kirkpatrick R, Zhang GF, Johanson K, Liu R, Lago A, Hofmann G, Macarron R, de los Frailes M, Perez P, Krawiec J, Winkler J, Jaye M: Identification of novel isoformselective inhibitors within class I histone deacetylases. J Pharmacol Exp Ther 2003, 307(2):720-728.

10. Minucci S, Pelicci PG: Histone deacetylase inhibitors and the promise of epigenetic (and more) treatments for cancer. Nat Rev Cancer 2006, 6(I):38-5I.

II. Finnin MS, Donigian JR, Cohen A, Richon VM, Rifkind RA, Marks PA, Breslow R, Pavletich NP: Structures of a histone deacetylase homologue bound to the TSA and SAHA inhibitors. Nature I999, 40 I(6749): | 88-193.

12. Acharya MR, Sparreboom A, Venitz J, Figg WD: Rational Development of Histone Deacetylase Inhibitors as Anti-cancer Agents: A Review. Mol Pharmacol 2005.

13. Phiel CJ, Zhang F, Huang EY, Guenther MG, Lazar MA, Klein PS: Histone deacetylase is a direct target of valproic acid, a potent anticonvulsant, mood stabilizer, and teratogen. J Biol Chem 200I, 276(39):36734-3674I.

14. Blanchard F, Chipoy C: Histone deacetylase inhibitors: new drugs for the treatment of inflammatory diseases? Drug Discov Today 2005, I0(3): 197-204.

15. McKinsey TA, Olson EN: Cardiac histone acetylation--therapeutic opportunities abound. Trends Genet 2004, 20(4):206-2I 3.

16. Qiu L, Burgess A, Fairlie DP, Leonard H, Parsons PG, Gabrielli BG Histone deacetylase inhibitors trigger a $\mathbf{G} 2$ checkpoint in normal cells that is defective in tumor cells. Mol Biol Cell 2000, I I(6):2069-2083

17. Burgess A, Ruefli A, Beamish H, Warrener R, Saunders N, Johnstone $R$, Gabrielli B: Histone deacetylase inhibitors specifically kill nonproliferating tumour cells. Oncogene 2004 23(40):6693-670I.

18. Schroeder TM, Westendorf J]: Histone deacetylase inhibitors promote osteoblast maturation. I Bone Miner Res 2005 20( I 2):2254-2263.

19. Iwami K, Moriyama T: Effects of short chain fatty acid, sodium butyrate, on osteoblastic cells and osteoclastic cells. Int J Biochem 1993, 25(I I): 1631-1635.

20. Sakata R, Minami S, Sowa Y, Yoshida M, Tamaki T: Trichostatin A activates the osteopontin gene promoter through API site. Biochem Biophys Res Commun 2004, 3 I 5(4):959-963.

2I. Jeon EJ, Lee KY, Choi NS, Lee MH, Kim HN, Jin YH, Ryoo HM, Choi JY, Yoshida M, Nishino N, Oh BC, Lee KS, Lee YH, Bae SC: Bone morphogenetic protein-2 stimulates Runx2 acetylation. J Biol Chem 2006, 28I(24): I6502-I65II.

22. Lee HW, Suh JH, Kim AY, Lee YS, Park SY, Kim JB: Histone deacetylase I-mediated histone modification regulates osteoblast differentiation. Mol Endocrinol 2006, 20( I 0):2432-2443.

23. Smith E, Frenkel B: Glucocorticoids inhibit the transcriptional activity of LEF/TCF in differentiating osteoblasts in a glycogen synthase kinase-3beta-dependent and -independent manner. $J$ Biol Chem 2005, 280(3):2388-2394.

24. Cho HH, Park HT, Kim YJ, Bae YC, Suh KT, Jung JS: Induction of osteogenic differentiation of human mesenchymal stem cells by histone deacetylase inhibitors. J Cell Biochem 2005.
25. Boer JD, Licht R, Bongers M, Klundert TV, Arends R, Blitterswijk CV: Inhibition of Histone Acetylation as a Tool in Bone Tissue Engineering. Tissue Eng 2006.

26. Schroeder TM, Kahler RA, Li X, Westendorf J]: Histone deacetylase 3 interacts with runx2 to repress the osteocalcin promoter and regulate osteoblast differentiation. I Biol Chem 2004, 279(40):41998-42007.

27. Edgar R, Domrachev M, Lash AE: Gene Expression Omnibus: NCBI gene expression and hybridization array data repository. Nucleic Acids Res 2002, 30(I):207-2 I0.

28. Qin L, Tamasi J, Raggatt L, Li X, Feyen JH, Lee DC, Dicicco-Bloom E, Partridge NC: Amphiregulin is a novel growth factor involved in normal bone development and in the cellular response to parathyroid hormone stimulation. I Biol Chem 2005, 280(5):3974-398I.

29. Roman-Roman S, Shi DL, Stiot V, Hay E, Vayssiere B, Garcia T, Baron R, Rawadi G: Murine Frizzled-I behaves as an antagonist of the canonical Wnt/beta-catenin signaling. J Biol Chem 2004, 279(7):5725-5733.

30. Bretscher A, Chambers D, Nguyen R, Reczek D: ERM-Merlin and EBP50 protein families in plasma membrane organization and function. Annu Rev Cell Dev Biol 2000, I 6: I I 3- I 43.

31. Weinman EJ, Hall RA, Friedman PA, Liu-Chen LY, Shenolikar S: The Association Of Nherf Adaptor Proteins With G ProteinCoupled Receptors And Receptor Tyrosine Kinases. Annu Rev Physiol 2006, 68:49I-505.

32. Sneddon WB, Syme CA, Bisello A, Magyar CE, Rochdi MD, Parent JL, Weinman EJ, Abou-Samra AB, Friedman PA: Activation-independent parathyroid hormone receptor internalization is regulated by NHERFI (EBP50). J Biol Chem 2003, 278(44):43787-43796.

33. Shibata T, Chuma M, Kokubu A, Sakamoto M, Hirohashi S: EBP50, a beta-catenin-associating protein, enhances Wnt signaling and is over-expressed in hepatocellular carcinoma. Hepatology 2003, 38(I): I 78- I86.

34. Shenolikar S, Voltz JW, Minkoff CM, Wade JB, Weinman EJ: Targeted disruption of the mouse NHERF-I gene promotes internalization of proximal tubule sodium-phosphate cotransporter type Ila and renal phosphate wasting. Proc Natl Acad Sci U S A 2002, 99(I 7): I | 470- I |475.

35. Glickman $\mathrm{MH}, \mathrm{Ciechanover} \mathrm{A}$ : The ubiquitin-proteasome proteolytic pathway: destruction for the sake of construction. Physiol Rev 2002, 82(2):373-428.

36. Garrett IR, Chen D, Gutierrez G, Zhao M, Escobedo A, Rossini G, Harris SE, Gallwitz W, Kim KB, Hu S, Crews CM, Mundy GR: Selective inhibitors of the osteoblast proteasome stimulate bone formation in vivo and in vitro. I Clin Invest 2003, I I I(I I): I77|-I782.

37. Zhao M, Qiao M, Harris SE, Oyajobi BO, Mundy GR, Chen D: Smurf I inhibits osteoblast differentiation and bone formation in vitro and in vivo. J Biol Chem 2004, 279( I 3): | 2854- I 2859.

38. Xu Q, Wang Y, Dabdoub A, Smallwood PM, Williams J, Woods C, Kelley MW, Jiang L, Tasman W, Zhang K, Nathans J: Vascular development in the retina and inner ear: control by Norrin and Frizzled-4, a high-affinity ligand-receptor pair. Cell 2004, I | 6(6):883-895.

39. Westendorf J, Kahler RA, Schroeder TM: Wnt signaling in osteoblasts and bone diseases. Gene 2004, 34 I:19-39.

40. Irizarry RA, Hobbs B, Collin F, Beazer-Barclay YD, Antonellis KJ, Scherf U, Speed TP: Exploration, normalization, and summaries of high density oligonucleotide array probe level data. Biostatistics 2003, 4(2):249-264. 